\title{
Polycythemia vera in pregnancy represents a challenge for a multidisciplinary collaboration: A case report and literature review
}

\author{
ROXANA ELENA BOHILTEA ${ }^{1-3}$, EMILIA NICULESCU-MIZIL ${ }^{4}$, \\ BIANCA MARGARETA MIHAI ${ }^{2}$, FLORENTINA FURTUNESCU ${ }^{5}$, IONITA DUCU ${ }^{2}$, \\ OCTAVIAN MUNTEANU ${ }^{2,6}$, TIBERIU AUGUSTIN GEORGESCU ${ }^{7}$ and CORINA GRIGORIU ${ }^{1,2}$
}

\author{
${ }^{1}$ Department of Obstetrics and Gynecology, 'Carol Davila' University of Medicine and Pharmacy, 020021 Bucharest; \\ ${ }^{2}$ Department of Obstetrics and Gynecology, University Emergency Hospital Bucharest, 050098 Bucharest; \\ ${ }^{3}$ Department of Obstetrics and Gynecology, Life Memorial Hospital, 012244 Bucharest; ${ }^{4}$ Department of Hematology, 'Provita' \\ Medical Center, 011442 Bucharest; ${ }^{5}$ Department of Public Health and Management, Faculty of Medicine, 'Carol Davila' \\ University of Medicine and Pharmacy, 050463 Bucharest; ${ }^{6}$ Department of Anatomy, 'Carol Davila' \\ University of Medicine and Pharmacy, 020021 Bucharest; ${ }^{7}$ Department of Pathology, Faculty of Medicine, 'Carol \\ Davila' University of Medicine and Pharmacy, 050463 Bucharest, Romania
}

Received July 23, 2021; Accepted August 24, 2021

DOI: 10.3892/etm.2021.10941

\begin{abstract}
Polycythemia vera (PV) is a rare chronic myeloproliferative neoplasm which represents an additional thrombotic factor in pregnancy. PV may be difficult to diagnose, particularly as its incidence is extremely uncommon among young women. The main diagnostic method involves a bone marrow biopsy, and high hemoglobin and platelet counts are usually indicative of the condition, after excluding other more frequent pathologies. PV is associated with a high risk of thrombosis, particularly in pregnancy, and requires anti-platelet treatment. At present, only a limited number of PV cases in pregnancy have been reported in the literature, at least to the best of our knowledge, with the largest case series being a retrospective study that included 25 pregnancies in 15 women. The present study describes the case of a patient diagnosed with JAK2-positive PV and also discusses this rare condition with particular focus on the following: i) The management of PV in pregnancy along with the additional pathologies in this specific case; and ii) the particularities of the pregnancy course. By identifying women suffering from PV superimposed by other possible procoagulant factors and
\end{abstract}

Correspondence to: Dr Bianca Margareta Mihai, Department of Obstetrics and Gynecology, University Emergency Hospital Bucharest, Splaiul Independenței 169, 050098 Bucharest, Romania E-mail: bmmihai@gmail.com

Key words: polycythemia vera, thrombocytosis, interferon, pregnancy, still birth, thrombophilia, hyperhomocysteinemia, fetal growth restriction applying the latest standard in healthcare, fetal and maternal prognosis may be significantly improved.

\section{Introduction}

Polycythemia vera (PV; polycythemia rubra or maladie de Vaquez) is a chronic myeloproliferative disorder characterized by the clonal proliferation of myeloid cells, which leads to an increase in red-blood cell mass, increasing the risk of patients to develop thrombosis (1). PV is associated with a significant risk of developing thromboembolic events; long-term complications are represented by myelofibrosis or even leukemic transformation (2). PV affects all ages in all populations, it can occur in early adulthood, although it rarely affects children and adolescents $(3,4)$; the median age at diagnosis is 60 years (5). As regards sex, PV has a slightly higher incidence among males than females (2.8 vs. 1.3 cases $/ 100,000$ per year) (6) and a lower incidence in Japan compared to Europe and the United States (7). The incidence of PV exhibits a tendency to increase with age among women, from an incidence of $0.04 / 100,000$ cases in the age group of 20-34 years to an incidence of $0.25 / 100,000$ in the age group of 35-39 years (2). The life expectancy of patients with symptomatic untreated PV has been found to be $\sim 18$ months; however, in treated patients, the survival rate is $>13$ years $(8,9)$.

PV, similar to other Philadelphia chromosome-negative myeloproliferative disorders, is usually caused by more than one genetic mutation (10). Major progress was made when the JAK2 (JAK2V617F) mutation was demonstrated to be the cause of PV (11). Other theories concerning the pathogenesis of $\mathrm{PV}$ have also been suggested, including the loss of heterozygosity ( $\mathrm{LOH}$ ), with $\mathrm{LOH}$ on the short arm of chromosome 9 (9pLOH) being found in 50\% of cases of PV and represents the most common chromosomal lesion as a 
result of mitotic recombination $(12,13)$. Another mechanism is family inheritance; there have been rare cases of familial PV reported, with PV exhibiting an autosomal dominant trait with incomplete penetrance. Clinical features in such cases present at a younger age compared with sporadic PV (14).

JAK/STAT signaling is responsible for cellular proliferation, as well as for cell survival, particularly via erythropoietin-erythropoietin receptor gene implicated in erythropoiesis. JAK2 mutation allows tyrosine kinase activity to continue the STAT phosphorylation activity in the presence of minimal or no erythropoietin levels $(15,16)$. In rare JAK2-negative PV cases, other JAK2 mutations have been identified in exon 12 (17). These mutations are heterogenous and they are sometimes difficult to detect by DNA sequencing or other conventional techniques due to the low allele burden (18).

Pregnant patients with PV are at a high risk of miscarriage, as well as other pregnancy-related complications, such as pregnancy-induced hypertension, intrauterine growth restriction, preeclampsia and placental abruption (19). The focus of treatment in pregnant women with PV is to maintain hematocrit levels $<45 \%$ or within the normal pregnancy hematocrit range (30-39\%), depending on which levels are lower (20). The preferred treatment among cytoreductive agents in fertile women or in pregnant women is interferon- $\alpha$ due to the teratogenic potential of the other agents, such as hydroxyurea, alkylating agents or ruxolitinib (21). As a complementary treatment, low-dose aspirin is strongly recommended in the management of patients with PV (22).

The present study describes the case of a 38-year-old pregnant woman with JAK2-positive masked PV diagnosed according to the World Health Organization (WHO) criteria (23) and associated pathologies and discusses this condition with an aim to highlight the following: i) Points of strategy for the management of PV during pregnancy; and ii) the particularities of the course of pregnancy in the case of PV.

\section{Case report}

The present study was conducted in accordance with the World Medical Association Declaration of Helsinki and was approved by the Ethical Board of the 'Life Memorial Hospital' Bucharest, Romania. The patient provided informed consent for publication of the present case report.

A 38-year-old woman presented to the authors' medical unit, Life Memorial Hospital (Bucharest, Romania), for an early diagnosis of pregnancy. The patient was diagnosed with JAK2-positive masked PV 3 years prior; the patient's medical history included a diagnosis of stage IV peripheral venous insufficiency, hepatic hemangioma, accessory spleen, hyperhomocysteinemia and hereditary thrombophilia (MTHFR C667T homozygote positive mutation).

The patient's haematological journey began at the age of 28 , when she was diagnosed with thrombocytosis [platelet (PLT) count, 600,000-700,000/ $\mu 1]$ with no other modified blood tests, which was considered reactive until 2014 when the patient was diagnosed with a JAK2-positive myeloproliferative neoplasm with essential thrombocythemia (PLT count, 700,000/ $\mu 1$ ); however, the patient refused to undergo a bone marrow biopsy.
In 2015, the patient presented to a medical unit complaining of vertigo and spontaneous phosphenes. A hemogram revealed 616,000 thrombocytes $/ \mu 1$, hemoglobin ( $\mathrm{Hb}$ ) levels of $14.8 \mathrm{~g} / \mathrm{dl}$, hematocrit (Ht) levels at $44.7 \%$ and a lymphocyte count of $10,720 / \mu 1$. After being subjected to several tests, the patient was diagnosed with hyperhomocysteinemia in the context of a MTFR C667T homozygote-positive mutation, marked poikilocytosis, ovalocytes, teardrop erythrocytes and rouleaux formation. The bone marrow biopsy revealed megakaryocyte hyperplasia with a secondary aspect. Serum erythropoietin or leucocyte alkaline phosphatase levels were not tested. Therefore, the patient was considered to have essential thrombocythemia (ET). The patient received treatment consisting of low-dose aspirin (150 mg daily), alopurinol, folic acid and Diosminum. In 2016, during a specialized control, due to further alterations in blood tests results (Hb levels, 17 g/dl; Ht, 51.4\%; lymphocyte count, 12,990/ $\mu$; PLT count, $760,000 / \mu 1)$, the patient was advised to commence interferon or hydroxyurea therapy; however, she refused and continued treatment with low-dose aspirin, Diosminum and folic acid. The following year, the patient's blood tests revealed further modifications: Hb levels, $18.3 \mathrm{~g} / \mathrm{dl}$; Ht, 53.3\%; lymphocyte count, 26,500/ $\mu \mathrm{l}$; PLT count, 1,048,000/ $\mu 1$, leucocyte alkaline phosphatase score of 42; and serum erythropoietin levels, $1.98 \mathrm{mU} / \mathrm{ml}$. Another bone marrow examination was performed and revealed panmyelosis, a small reactive lymphoid nodule, CD34+ cells $(2-3 \%)$ and sinus dilation, suggestive of PV. The patient accepted immunomodulatory treatment (3 MU pegylated interferon- $\alpha$ subcutaneously administered three times/week), a short time before she became pregnant. From her family medical history, it was noted that her father was suffering from insulin-dependent diabetes mellitus and renal cancer. Her gynecological anamnesis consisted of an abortion with no further complications.

The ultrasound evaluation of the incipient pregnancy was normal for the gestational age. The patient was counseled about the possible risks and complications during pregnancy, considering the associated hematological pathology and received the recommendation for proper hydration and to wear compression stockings. During the pregnancy, an obstetrician and hematologist closely monitored the patient, and anti-coagulant treatment (4,000 UI enoxaparin/day) was introduced in addition to the patient's previous medication, considering the cumulative thrombotic risk due to pregnancy, thrombocytosis, chronic venous disease and the advanced maternal age. The patient was subjected to a non-invasive prenatal screening test for the major chromosomal abnormalities, the result of which was negative. Due to the favorable evolution of PV, consisting of the normalization of the hemogram and particularly of the thrombocyte value (Fig. 1), the cytoreductive treatment was reduced to two administrations per week of pegylated interferon- $\alpha$, commencing on the 23th week of gestation.

The clinical and ultrasound evaluation during pregnancy revealed a normal fetal growth and a normal amniotic fluid index. There were no thrombotic events and the thrombocyte count exhibited an unexpected decreasing trend, being maintained within safety limits during the entire pregnancy. The pregnancy was finalized at 38 weeks and 5 days and a male fetus weighing 3,190 g, with an Apgar score of 8 at $1 \mathrm{~min}$, was delivered by cesarean section, with a slightly poor adaptation 


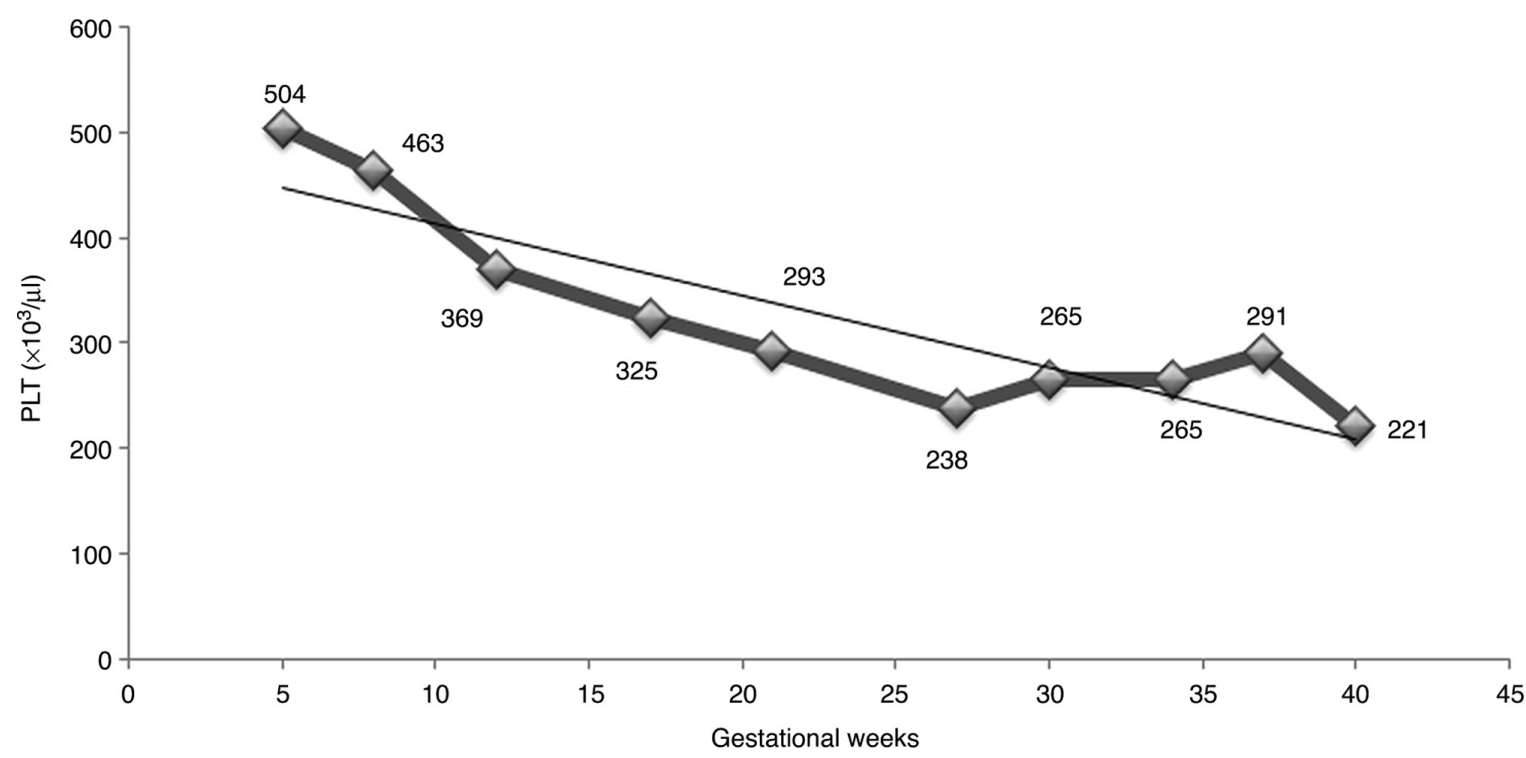

Figure 1. Dynamics of thrombocyte count during the pregnancy of the patient. PLT, platelet.

to the extra-uterine life. The further evolution of the offspring was favorable, with no other neonatal complications.

\section{Discussion}

The particularity of the case of PV in pregnancy presented herein embodies the difficulties in diagnosis of this pathology in a woman of fertile age, and the need for interdisciplinary collaboration in order to obtain a good and safe outcome for both mother and child. A young age for PV, but an advanced maternal age is the most challenging background for a high-risk pregnancy with multiple and complex associated diseases. The authors' experience in the management of myeloproliferative diseases was demonstrated in a previous study published 3 years ago (24); the pregnancy associated with primary myelofibrosis had a good outcome under combined treatment with interferon- $\alpha$ and low-dose aspirin, and even an improved hematological evolution marked by the same decrease in platelet counts following low molecular-weight heparin (LMWH) administration in the third trimester (24).

The evolution of latent PV with thrombocytosis mimicking essential thrombocythemia was previously described in a series of 23 cases of masked PV by Thiele et al (25), revealing the explanation for the long journey to the final diagnosis with four-field 2016 WHO criteria for essential thrombocythemia (26) and the special pattern of the second bone marrow histopathology of the patient described herein.

The preferred drug in the initial treatment of PV is hydroxyurea, as a result of its efficacy, low-cost, comfort in administration and limited associated toxicity (27-29). However, for young patients $(<40$ years of age) and pregnant patients, the elective drug is interferon- $\alpha$ due to its possibility in achieving cytogenetic remission and its safety in pregnancy in contrast to the teratogenicity of hydroxyurea (20). Interferon- $\alpha$ therapy is quite a relatively recent acquisition in the treatment of myeloproliferative disorders, exhibiting its effectiveness in normalizing the platelet count (30). The fetal safety of interferon- $\alpha$ was previously investigated by
Yazdani Brojeni et al (31), who concluded that interferon- $\alpha$ does not significantly increase the risk of major malformation, spontaneous abortion, fetal demise, or preterm delivery when compared with the general population rate.

To the best of our knowledge, there are limited studies available in the literature debating the management of PV in pregnancy. A recent study by Robinson et al (2) concluded that it is essential for the optimal fetal prognosis to act actively in the hemogram balance. The aspirin administration is mainly based on the results of the European Collaboration on Low-Dose Aspirin in Polycythemia Vera (ECLAP) trial, which demonstrated that $100 \mathrm{mg}$ aspirin daily reduced the risk of the combined endpoint of non-fatal myocardial infarction, non-fatal stroke, pulmonary embolism, major venous thrombosis, or death from cardiovascular causes (relative risk, 0.40; 95\% CI 0.18-0.91) (22). The mechanism of the efficacy of low-dose aspirin is the irreversible inhibition of the enzyme, COX-1, in the vascular endothelium, necessary for the production of thromboxane A2, the factor responsible for platelet aggregation and vasoconstriction. As regards the administration of LMWH, it is not prophylactically recommended for women without active thrombosis or a history of a major thrombotic event $(20,29,30)$. For the case in the present study, the use of a $150 \mathrm{mg}$ daily dose of aspirin was considered, based on the authors' experience in screening and preventing, first and foremost preeclampsia, but also other fetal and maternal complications and the associated risks of intrauterine growth restriction, premature rupture of membranes, abruptio placentae and intrauterine fetal death $(32,33)$. The justification in adding enoxaparin to the treatment of the patient was the existence of associated pathologies: Peripheral venous insufficiency with a high risk of thrombosis in the context of pregnancy and thrombocytosis, as well as an advanced maternal age. Thus, it can be confirmed that better results are obtained when using cytoreductive treatment and low-dose aspirin; the use of anti-coagulant treatment should be individualized and recommended whenever needed. 
In conclusion, pregnancy by definition generates a hypercoagulability status; PV represents a procoagulant factor due to the elevated thrombocyte count, and due to a high risk of thrombosis, thrombophilia screening is recommended, particularly in PV, for each and every pregnant women or women who are aiming at conceiving. In addition, the active implication and management of this pathology is required in order to improve live births.

\section{Acknowledgements}

Not applicable.

\section{Funding}

No funding was received.

\section{Availability of data and materials}

All data generated or analyzed during this study are included in this published article.

\section{Authors' contributions}

REB conceived the article following the successful management of the presented case. BMM and ID performed the literature search and wrote the manuscript. TAG, FF, OM and $\mathrm{CG}$ contributed to the literature review. ENM conducted the follow-up of the patient. REB, ENM, CG and FF collected, assembled and interpreted the data, and revised the manuscript critically for important intellectual content. REB and BMM confirm the authenticity of all the raw data. All authors have read and approved the final manuscript.

\section{Ethics approval and consent to participate}

The present study was conducted in accordance with the World Medical Association Declaration of Helsinki and was approved by the Ethical Board of the 'Life Memorial Hospital' Bucharest, Romania.

\section{Patient consent for publication}

The patient provided informed consent for publication of the present case report.

\section{Competing interests}

The authors declare that they have no competing interests.

\section{References}

1. Stuart BJ and Viera AJ: Polycythemia vera. Am Fam Physician 69 : 2139-2144, 2004.

2. Robinson S, Bewley S, Hunt BJ, Radia DH and Harrison CN The management and outcome of 18 pregnancies in women with polycythemia vera. Haematologica 90: 1477-1483, 2005.

3. Passamonti F, Malabarba L, Orlandi E, BaratèC, Canevari A, Brusamolino E, Bonfichi M, Arcaini L, Caberlon S, Pascutto C and Lazzarino M: Polycythemia vera in young patients: A study on the long-term risk of thrombosis, myelofibrosis and leukemia. Haematologica 88: 13-18, 2003.
4. Teofili L, Giona F, Martini M, Cenci T, Guidi F, Torti L, Palumbo G, Amendola A, Foà R and Larocca LM: Markers of myeloproliferative diseases in childhood polycythemia vera and essential thrombocythemia. J Clin Oncol 25: 1048-1053, 2007.

5. Tefferi A, Rumi E, Finazzi G, Gisslinger H, Vannucchi AM, Rodeghiero F, Randi ML, Vaidya R, Cazzola M, Rambaldi A, et al: Survival and prognosis among 1545 patients with contemporary polycythemia vera: An international study. Leukemia 27: 1874-1881, 2013.

6. Anía BJ, Suman VJ, Sobell JL, Codd MB, Silverstein MN and Melton LJ III: Trends in the incidence of polycythemia vera among olmsted county, Minnesota residents, 1935-1989. Am J Hematol 47: 89-93, 1994.

7. Johansson P: Epidemiology of the myeloproliferative disorders polycythemia vera and essential thrombocythemia. Semin Thromb Hemost 32: 171-173, 2006.

8. Chievitz E and Thiede T: Complications and causes of death in polycythaemia vera. Acta Med Scand 172: 513-523, 1962.

9. Tefferi A, Guglielmelli P, Larson DR, Finke C, Wassie EA, Pieri L, Gangat N, Fjerza R, Belachew AA, Lasho TL, et al: Long-term survival and blast transformation in molecularly annotated essential thrombocythemia, polycythemia vera, and myelofibrosis. Blood 124: 2507-2513, 2014.

10. Campbell PJ and Green AR: The myeloproliferative disorders. N Engl J Med 355: 2452-2466, 2006.

11. Schafer AI: Molecular basis of the diagnosis and treatment of polycythemia vera and essential thrombocythemia. Blood 107: 4214-4222, 2006.

12. Kralovics R, Buser AS, Teo SS, Coers J, Tichelli A, van der Maas AP and Skoda RC: Comparison of molecular markers in a cohort of patients with chronic myeloproliferative disorders. Blood 102: 1869-1871, 2003.

13. Kralovics R, Guan Y and Prchal JT: Acquired uniparental disomy of chromosome $9 \mathrm{p}$ is a frequent stem cell defect in polycythemia vera. Exp Hematol 30: 229-236, 2002.

14. Kralovics R, Stockton DW and Prchal JT: Clonal hematopoiesis in familial polycythemia vera suggests the involvement of multiple mutational events in the early pathogenesis of the disease. Blood 102: 3793-3796, 2003.

15. Levy DE and Darnell JE Jr: Stats: Transcriptional control and biological impact. Nat Rev Mol Cell Biol 3: 651-662, 2002.

16. Neubauer H, Cumano A, Müller M, Wu H, Huffstadt U and Pfeffer K: Jak2 deficiency defines an essential developmental checkpoint in definitive hematopoiesis. Cell 93: 397-409, 1998.

17. Scott LM, Tong W, Levine RL, Scott MA, Beer PA, Stratton MR, Futreal PA, Erber WN, McMullin MF, Harrison CN, et al: JAK2 exon 12 mutations in polycythemia vera and idiopathic erythrocytosis. N Engl J Med 356: 459-468, 2007.

18. Scott LM: The JAK2 exon 12 mutations: A comprehensive review. Am J Hematol 86: 668-676, 2011.

19. Ferguson JE II, Ueland K and Aronson WJ: Polycythemia rubra vera and pregnancy. Obstet Gynecol 62 (Suppl 3): 16S-20S, 1983.

20. Barbui T, Barosi G, Birgegard G, Cervantes F, Finazzi G, Griesshammer M, Harrison C, Hasselbalch HC, Hehlmann R, Hoffman R, et al: Philadelphia-negative classical myeloproliferative neoplasms: Critical concepts and management recommendations from European LeukemiaNet. J Clin Oncol 29: 761-770, 2011.

21. Harrison C: Pregnancy and its management in the Philadelphia negative myeloproliferative diseases. Br J Haematol 129: 293-306, 2005.

22. Landolfi R, Marchioli R, Kutti J, Gisslinger H, Tognoni G, Patrono $\mathrm{C}$ and Barbui T; European Collaboration on Low-Dose Aspirin in Polycythemia Vera Investigators: Efficacy and safety of low-dose aspirin in polycythemia vera. N Engl J Med 350: 114-124, 2004

23. Barbui T, Thiele J, Gisslinger H, Kvasnicka HM, Vannucchi AM, Guglielmelli P, Orazi A and Tefferi A: The 2016 WHO classification and diagnostic criteria for myeloproliferative neoplasms: Document summary and in-depth discussion. Blood Cancer J 8: 15, 2018.

24. Bohîlţea RE, Cîrstoiu MM, Ionescu CA, Niculescu-Mizil E, Vlădăreanu AM, Voican I, Dimitriu M and Turcan N: Primary myelofibrosis and pregnancy outcomes after low molecular-weight heparin administration: A case report and literature review. Medicine (Baltimore) 96: e8735, 2017. 
25. Thiele J, Kvasnicka HM and Diehl V: Initial (latent) polycythemia vera with thrombocytosis mimicking essential thrombocythemia. Acta Haematol 113: 213-219, 2005.

26. Arber DA, Orazi A, Hasserjian R, Thiele J, Borowitz MJ, Le Beau MM, Bloomfield CD, Cazzola M and Vardiman JW: The 2016 revision to the World Health Organization classification of myeloid neoplasms and acute leukemia. Blood 127: 2391-2405, 2016.

27. Beauverd Y, Radia D, Cargo C, Knapper S, Drummond M, Pillai A, Harrison C and Robinson S: Pegylated interferon alpha-2a for essential thrombocythemia during pregnancy: Outcome and safety. A case series. Haematologica 101: e182-e184, 2016

28. Finazzi $\mathrm{G}$ and Barbui T: How I treat patients with polycythemia vera. Blood 109: 5104-5111, 2007.

29. Fruchtman SM, Mack K, Kaplan ME, Peterson P, Berk PD and Wasserman LR: From efficacy to safety: A polycythemia vera study group report on hydroxyurea in patients with polycythemia vera. Semin Hematol 34: 17-23, 1997.
30. Bertozzi I, Rumi E, Cavalloni C, Cazzola M, Fabris F and Randi ML: Pregnancy outcome and management of 25 pregnancies in women with polycythemia vera. Am J Hematol 93: E234-E235, 2018.

31. Yazdani Brojeni P, Matok I, Garcia Bournissen F and Koren G: A systematic review of the fetal safety of interferon alpha. Reprod Toxicol 33: 265-268, 2012.

32. Bohiltea RE, Cirstoiu MM, Turcan N, Stoian AP, Zugravu CA, Munteanu O, Arsene LV, Oana B, Neacsu A and Furtunescu F: Inherited thrombophilia is significantly associated with severe preeclampsia. Exp Ther Med 21: 261-267, 2021

33. Grigoriu C, Anca AF, Grigoras M, Cezar C, Lungu A and Horhoianu V: Premature birth and systemic inflammatory response syndrome. Gineco Ro 4: 80-83, 2008. 